\title{
Expression of enoyl coenzyme A hydratase, short chain, 1, in colorectal cancer and its association with clinicopathological characteristics
}

\author{
JUN-PEI XIE ${ }^{1}, \mathrm{XIAO}^{-S A N} \mathrm{ZHU}^{1}$, YI-CHEN DAI ${ }^{1}, \mathrm{CUI} \mathrm{YU}^{2}$, TAO XIE $^{1}$ and ZHANG-XING CHEN $^{1}$ \\ ${ }^{1}$ Department of Gastroenterology, The Chenggong Hospital Affiliated to Xiamen University, Xiamen, Fujian 361003; \\ ${ }^{2}$ Department of Gastroenterology, The Second Hospital of Baoding City, Baoding, Hebei 071051, P.R. China
}

Received February 27, 2014; Accepted May 22, 2014

DOI: $10.3892 / \mathrm{mco} .2014 .340$

\begin{abstract}
This study aimed to investigate the expression and clinical significance of enoyl coenzyme A hydratase, short chain, 1 (ECHS1), in patients with colorectal cancer (CRC). The ECHS1 protein expression as detected by immunohistochemistry in 148 CRC specimens was evaluated and compared by clinical pathology and prognosis; 38 specimens from proximal non-cancerous colorectal tissues were included as controls. The ECHS1 protein expression was also measured by western blot analysis in 46 fresh CRC tissue specimens and 22 normal colorectal tissue specimens. The rate of positive ECHS1 expression differed significantly between the CRC tissues $(56.76 \%, 84 / 148)$ and the proximal non-cancerous colorectal tissues $(5.26 \%, 2 / 38)(\mathrm{P}<0.001)$. The ECHS1 protein expression was confirmed not to be associated with gender or age. However, the positive expression of ECHS1 tended to be positively associated with clinical TNM stage $(\mathrm{P}=0.015)$, lymph node metastasis $(\mathrm{P}=0.011)$ and histological differentiation $(\mathrm{P}=0.028)$. The expression of the ECHS1 protein on western blot analysis was significantly increased in CRC vs. normal tissues. In addition, the overall survival curves estimated with the Kaplan-Meier method demonstrated that CRC patients exhibiting low ECHS1 expression survived significantly longer compared to patients with high ECHS1 levels $(\mathrm{P}=0.039)$. Our data suggested that ECHS1 protein expression may contribute to the occurrence, progression and metastasis of CRC, is closely associated with prognosis and may provide useful information for CRC molecular-targeted therapy.
\end{abstract}

Correspondence to: Dr Xiao-San Zhu, Department of Gastroenterology, The Chenggong Hospital Affiliated to Xiamen University, 94-96 Wenyuan Road, Xiamen, Fujian 361003, P.R. China

E-mail: zhuxs223@126.com

Key words: colorectal cancer, enoyl coenzyme A hydratase, short chain, 1, metastasis, prognosis

\section{Introduction}

Colorectal cancer (CRC) is a major health concern worldwide and is one of the most frequent causes of cancer-related mortality (1). Despite the advances in diagnosis and treatment, $\sim 25 \%$ of CRC patients eventually succumb to their disease (2). The most common causes of treatment failure and/or death in patients with CRC are tumor invasion and metastasis (3). The mechanism underlying CRC development has not been clearly determined, although a series of genetic and epigenetic events are considered to be involved in colorectal carcinogenesis, including oncogene activation and anti-oncogene inactivation (4). The second step in the physiologically critical $\beta$-oxidation pathway of fatty acid metabolism in the mitochondria is catalyzed by enoyl coenzyme A hydratase, short chain, 1 (ECHS1), which is assigned to chromosome 10q26.2-q26.3 and promotes proliferation of several tumor cells, such as hepatocellular carcinoma cells (5). Yeh et al (6) reported that ECHS1 may play an important role in human colorectal carcinogenesis via microarray bioinformatics analysis. However, definite conclusions regarding the extent of ECHS1 expression in human CRCs and its significance have not yet been reached. In this study, we investigated the expression of ECHS1 by immunohistochemical analysis in human CRC samples and proximal non-cancerous colorectal tissues and its association with clinicopathological characteristics and prognosis. The expression of the ECHS1 protein in fresh CRC specimens and normal colorectal tissues was detected by western blot analysis.

\section{Materials and methods}

Specimens and clinicopathological data. We retrospectively collected samples from 148 patients who had undergone surgical treatment for pathologically confirmed primary CRC at the Chenggong Hospital Affiliated to Xiamen University, between March, 2010 and January, 2013 (Table I). None of the patients had received chemotherapy or radiotherapy prior to surgery. This study was conducted in accordance with the ethical principles of the Declaration of Helsinki and approved by the Review Committee for the Use of Human or Animal Subjects of Xiamen University. Informed consent was obtained 
Table I. Association between ECHS1 expression and clinicopathological characteristics in CRC and proximal non-cancerous colorectal tissues.

\begin{tabular}{|c|c|c|c|c|}
\hline \multirow[b]{2}{*}{ Variables } & \multirow[b]{2}{*}{$\mathrm{n}$} & \multicolumn{2}{|c|}{ ECHS1 } & \multirow[b]{2}{*}{ P-value } \\
\hline & & Negative & Positive & \\
\hline \multicolumn{5}{|l|}{ Tissue type } \\
\hline CRC & 148 & 64 & 84 & $<0.001$ \\
\hline Paraneoplastic & 38 & 36 & 2 & \\
\hline \multicolumn{5}{|l|}{ Age, years } \\
\hline$<55$ & 76 & 40 & 36 & 0.419 \\
\hline$\geq 55$ & 72 & 42 & 30 & \\
\hline \multicolumn{5}{|l|}{ Gender } \\
\hline Male & 78 & 38 & 40 & 0.623 \\
\hline Female & 70 & 36 & 34 & \\
\hline \multicolumn{5}{|l|}{ Differentiation } \\
\hline High/Moderate & 86 & 34 & 52 & 0.028 \\
\hline Low & 62 & 20 & 42 & \\
\hline Stage & & & & 0.015 \\
\hline $\mathrm{I}$ & 30 & 6 & 24 & \\
\hline II & 44 & 10 & 34 & \\
\hline III & 48 & 12 & 36 & \\
\hline IV & 26 & 4 & 22 & \\
\hline \multicolumn{5}{|l|}{ LN metastasis } \\
\hline No & 38 & 20 & 18 & 0.011 \\
\hline N1 & 54 & 24 & 30 & \\
\hline $\mathrm{N} 2$ & 56 & 14 & 42 & \\
\hline
\end{tabular}

ECHS1, enoyl coenzyme A hydratase, short chain, 1; CRC, colorectal cancer; LN, lymph node.

from all the participants. A total of 78 men and 70 women, with a median age of 51.5 years (range, 33-71 years), were included in this study. The post-surgery pathological reports confirmed that all the CRCs were adenocarcinomas. The median follow-up was 19 months (range, 10-26 months). According to the International Union Against Cancer-TNM stage, the patients were divided into 30 stage I, 44 stage II, 48 stage III and 26 stage IV cases. A total of 38 proximal non-cancerous colorectal tissues were included as controls. In addition, 46 fresh tissue samples of CRC and 22 normal colorectal tissues were collected for western blot analysis.

Immunohistochemistry and evaluation criteria. The specimens were fixed in $10 \%$ formalin and embedded in paraffin, according to standard procedures. Tissue sections $(4 \mu \mathrm{m})$ were prepared and endogenous peroxidase activity was blocked by incubation in $0.3 \% \mathrm{H}_{2} \mathrm{O}_{2}$ for $20 \mathrm{~min}$. The sections were incubated with rabbit anti-ECHS1 monoclonal antibody (1:400 dilution; Abcam, Cambridge, UK) overnight at $4^{\circ} \mathrm{C}$. The secondary antibody used was goat anti-rabbit $\operatorname{IgG}(1: 5,000$ dilution). The specimens were examined using the Envision kit (Dako Canada Inc., Burlington, ON, Canada) using diaminobenzidine as a chromogen.
The evaluation of the grade of staining was independently performed by an experienced pathologist. The criteria used for assessment were previously reported as follows: -, negative;,$+ 1-25 \%$ positive cells;,$++ 25-75 \%$ positive cells; and,$+++ 75-100 \%$ positive cells (7).

Western blot analysis. The proteins $(20 \mu \mathrm{g})$ were subjected to $10 \%$ SDS polyacrylamide gel electrophoresis and transferred onto polyvinylidene difluoride membranes (Millipore Corp., Billerica, MA, USA) using the Mini-Protean system (Bio-Rad, Hercules, CA, USA). The membranes were incubated for $1 \mathrm{~h}$ at room temperature in blocking buffer and then incubated with the appropriate antibodies (anti-ECHS1 antibody at 1:400 dilution, Abcam; and anti-tubulin antibody at 1:2,000 dilution, Santa Cruz Biotechnology Inc., Santa Cruz, CA, USA) overnight at $4^{\circ} \mathrm{C}$. After washing with Tris-buffered saline, Tween-20 and Triton X-100, the membranes were incubated with horseradish peroxidase-conjugated anti-rabbit antibody (1:8,000 dilution; ZSGB-BIO, Beijing, China) for $2 \mathrm{~h}$ at $37^{\circ} \mathrm{C}$. The blots were briefly incubated with enhanced chemiluminescence solution (Applygen Technologies, Inc., Beijing, China) and exposed to X-ray films.

Statistical analysis. Statistical analyses were performed using SPSS.17.0 software (SPSS Inc., Chicago, IL, USA). The Pearson's Chi-square test was used for recepteur d'origine nantais expression positive rate comparison. The Kaplan-Meier method was used for evaluating survival curves and the long-rank test was used for testing survival rates. $\mathrm{P}<0.05$ was considered to indicate a statistically significant difference.

\section{Results}

Expression of ECHS1 protein in CRC and proximal non-cancerous colorectal tissues. ECHS1 protein expression was detected in the cytoplasm of CRC cells (Fig. 1). The percentage of positively stained carcinoma cells and the staining intensity were recorded. The positive expression rate of the ECHS1 was $56.76 \%(84 / 148)$ in CRC cases and only $5.26 \%(2 / 38)$ in proximal non-cancerous colorectal tissues. The difference between the two groups was statistically significant $\left(\chi^{2}=13.06 ; \mathrm{P}<0.001\right)$ (Table I).

ECHS1 protein expression in fresh tissues. The expression of the ECHS1 protein in 46 fresh CRC and 22 normal colorectal tissue samples was detected by western blot analysis. We observed that the expression level of the ECHS1 protein in fresh CRC tissues was significantly higher compared to that in normal colorectal tissues. Furthermore, the expression level of the ECHS1 protein in fresh samples of poorly differentiated CRC was significantly higher compared to that in CRC samples of high or moderate differentiation (Fig. 2A and B).

Association between ECHS1 expression and clinicopathological characteristics. The ECHS1 protein was strongly expressed in CRC tissues classified as the high TNM stage group, positive lymph node metastasis group and poorly differentiated group (Fig. 1 and Table I). The expression of the ECHS1 protein was positively correlated with clinical TNM stage $\left(\chi^{2}=10.46 ; P=0.015\right)$, lymph node metastasis $\left(\chi^{2}=7.93\right.$; 
A

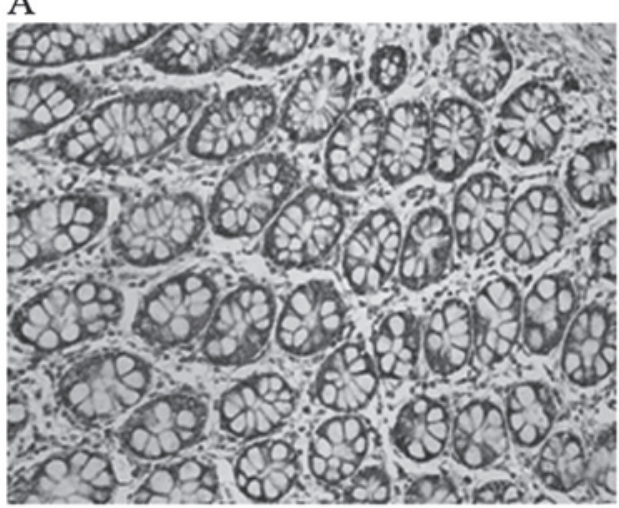

$\mathrm{C}$

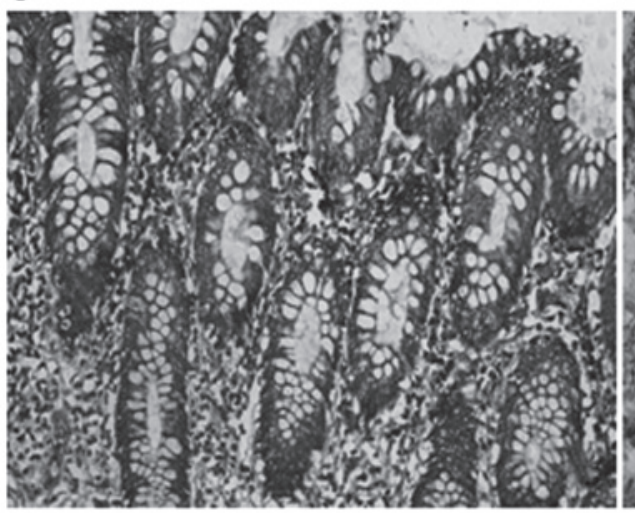

B

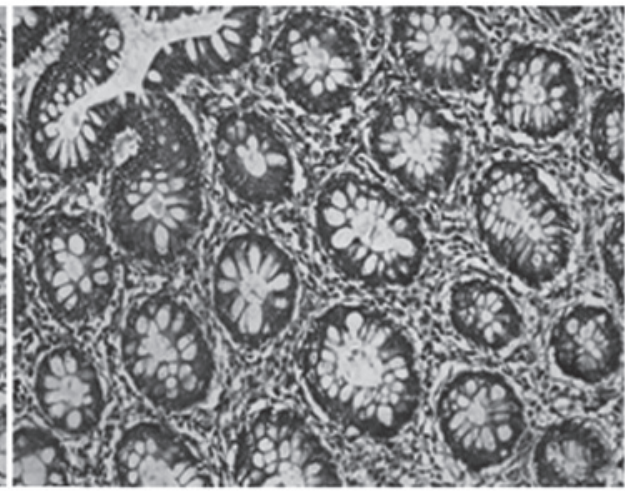

$\mathrm{D}$

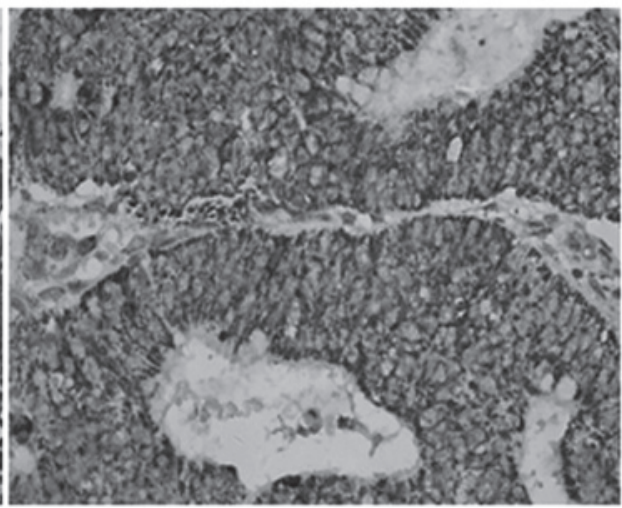

Figure 1. Detection of enoyl coenzyme A hydratase, short chain, 1 expression in colorectal cancer and paraneoplastic tissues by immunohistochemistry (diaminobenzidine; original magnification, x200). (A) Paraneoplastic tissues. (B) Highly differentiated adenocarcinoma. (C) Moderately differentiated adenocarcinoma. (D) Poorly differentiated adenocarcinoma.
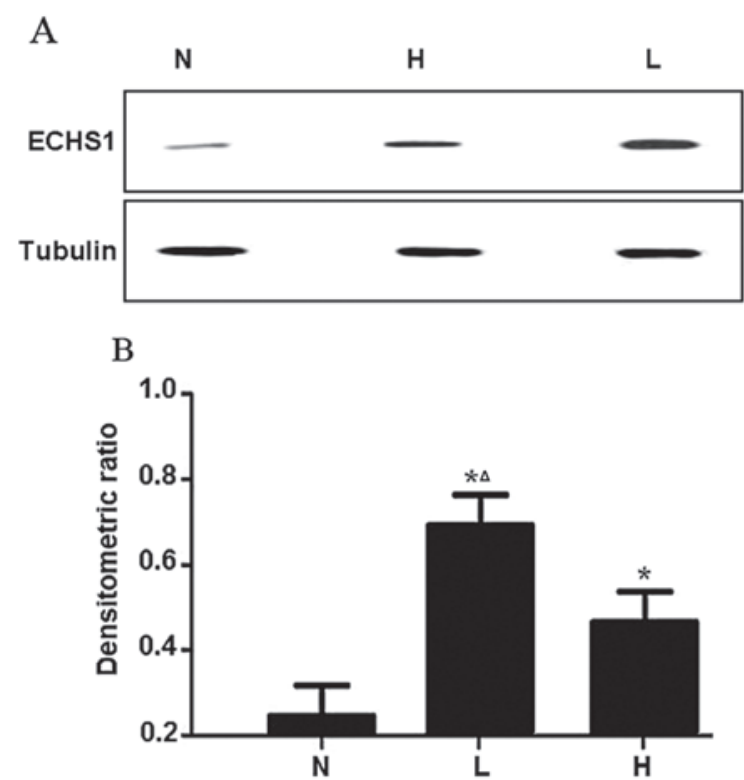

Figure 2. Western blot analysis of enoyl coenzyme A hydratase, short chain, 1 (ECHS1), and tubulin for lysates of normal colorectal tissues (N) and colorectal cancer tissues (L, low differentiation; H, high differentiation). Tubulin was used as a loading control. The data are presented as means \pm standard error of the mean. ${ }^{*} \mathrm{P}<0.05$ vs. $\mathrm{N} ;{ }^{\Delta} \mathrm{P}<0.05$ vs. $\mathrm{H}$.

$\mathrm{P}=0.011)$ and histological differentiation $\left(\chi^{2}=5.73 ; \mathrm{P}=0.024\right)$ with statistically significance differences, which was not the case for gender or age $(\mathrm{P}>0.05)$ (Table I).

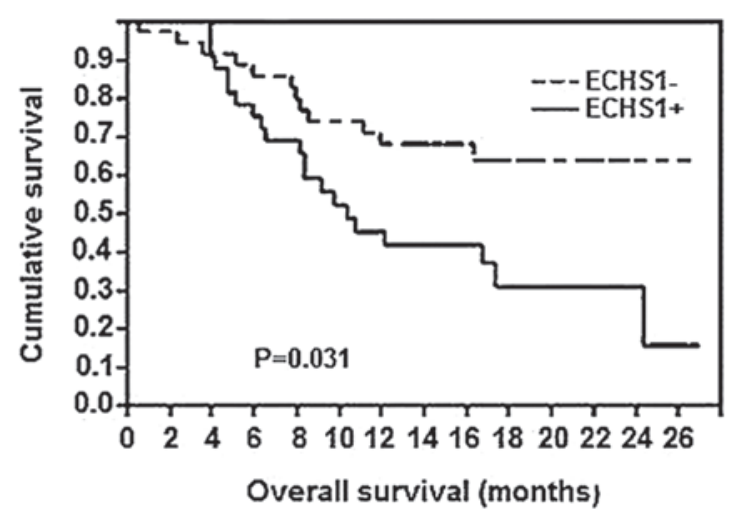

Figure 3. Kaplan-Meier survival curve for positive and negative enoyl coenzyme A hydratase, short chain, 1 (ECHS1), expression in colorectal cancer patients. $\mathrm{P}=0.031$, log-rank test.

ECHS1 expression and survival. Of the 148 CRC patients, 110 patients remained alive at the time of this study, whereas 34 patients had succumbed to distant metastases and 4 patients had succumbed to locoregional disease recurrence. As regards the Kaplan-Meier survival analysis (Fig. 3), a significant difference in the overall survival rate was observed between patients with positive expression of the ECHS1 protein and those with negative ECHS1 expression $(\mathrm{P}=0.031)$. Therefore, the expression of the ECHS1 protein was associated with a poor prognosis in patients with CRC. 


\section{Discussion}

Under physiological conditions, ECHS1 is essential for embryonic development and is critical in the regulation of certain physiological processes $(8,9)$. Inappropriate activation or altered expression of ECHS1 is involved in several regulatory processes associated with the occurrence and progression of tumors. Elevated expression of the ECHS1 protein has been detected in several malignant tumors, including liver, colorectal, breast, bladder and prostate cancers (10). In this study, we observed a significant increase in the expression of the ECHS1 protein in CRC tissues compared to that in proximal non-cancerous colorectal tissues. The western blot analysis confirmed that the expression of the ECHS1 protein was significantly increased in CRC vs. normal tissues, indicating that ECHS1 may be involved in the development of CRC and inferring that ECHS1 may be a novel tumor marker for CRC.

Through the analysis of the association between the expression of ECHS1 and clinicopathological characteristics, we demonstrated that the positive expression rate of the ECHS1 protein in CRC was correlated with the TNM stage (11-13). Lymphogenous metastases are usually an early step during cancer progression, with lymph nodes commonly being the first metastatic site $(13,14)$. The presence of lymph node metastases is known to be associated with poor prognosis in a number of tumors $(15,16)$. Lymph node metastasis and histological differentiation were also found to be correlated with ECHS1 protein expression. These data suggest that ECHS1 may be involved in the progression, invasion and metastasis of CRC. Previous studies on ECHS1 expression and prognostic significance demonstrated that elevated ECHS1 expression was associated with a significantly poorer prognosis in several types of tumors, including breast, liver and colorectal cancers $(5,6,9)$.

Of note, we observed that the expression of the ECHS1 protein was positively correlated with histological differentiation. The ECHS1 protein expression was significantly higher in poorly differentiated CRC tissues compared to that in CRC tissues of high or moderate differentiation. In our study, there was also a significant association between the expression of the ECHS1 protein and the overall survival rate. These findings suggest that positive ECHS1 expression may be useful as a novel prognostic marker in CRC patients and a potential target for therapeutic intervention.

\section{Acknowledgements}

This study was supported by grants from the Scientific Research Foundation of Health Bureau of Xiamen City (no. 3502Z20134025) and the Medical Technology Innovation Project of Nanjing Military (no. MS090), P.R. China.

\section{References}

1. Fehlker M, Huska MR, Jöns T, et al: Concerted down-regulation of immune-system related genes predicts metastasis in colorectal carcinoma. BMC Cancer 14: 64, 2014

2. Debunne $\mathrm{H}$ and Ceelen W: Mucinous differentiation in colorectal cancer: molecular, histological and clinical aspects. Acta Chir Belg 113: 385-390, 2013.

3. Chen YS, Xu SX, Ding YB, et al: Helicobacter pylori infection and the risk of colorectal adenoma and adenocarcinoma: an updated meta-analysis of different testing methods. Asian Pac J Cancer Prev 14: 7613-7619, 2013.

4. Ewing I, Hurley JJ, Josephides E, et al: The molecular genetics of colorectal cancer. Frontline Gastroenterol 5: 26-30, 2014.

5. Zhu XS, Dai YC, Chen ZX, et al: Knockdown of ECHS1 protein expression inhibits hepatocellular carcinoma cell proliferation via suppression of Akt activity. Crit Rev Eukaryot Gene Expr 23: 275-282, 2013.

6. Yeh CS, Wang JY, Cheng TL, et al: Fatty acid metabolism pathway play an important role in carcinogenesis of human colorectal cancers by microarray-bioinformatics analysis. Cancer Lett 233: 297-308, 2006.

7. Wu CW, Li AF, Chi CW, et al: Human gastric cancer kinase profile and prognostic significance of MKK4 kinase. Am J Pathol 156: 2007-2015, 2000.

8. De Preter V, Arijs I, Windey K, et al: Impaired butyrate oxidation in ulcerative colitis is due to decreased butyrate uptake and a defect in the oxidation pathway. Inflamm Bowel Dis 18: 1127-1136, 2012.

9. Liu X, Feng R and Du L: The role of enoyl-CoA hydratase short chain 1 and peroxiredoxin 3 in PP2-induced apoptosis in human breast cancer MCF-7 cells. FEBS Lett 584: 3185-3892, 2010.

10. Resch A and Langner C: Lymph node staging in colorectal cancer: old controversies and recent advances. World J Gastroenterol 19: 8515-8526, 2013.

11. Lou X, Qi X, Zhang Y, Long H and Yang J: Decreased expression of microRNA-625 is associated with tumor metastasis and poor prognosis in patients with colorectal cancer. J Surg Oncol 108: 230-235, 2013.

12. Miglio U, Mezzapelle R, Paganotti A, et al: Mutation analysis of KRAS in primary colorectal cancer and matched metastases by means of highly sensitivity molecular assay. Pathol Res Pract 209: 233-236, 2013.

13. Kawamata F, Homma S, Kamachi H, et al: C-ERC/mesothelin provokes lymphatic invasion of colorectal adenocarcinoma. J Gastroenterol 49: 81-92, 2014.

14. Jiang B, Mason J, Jewett A, et al: Cell budding from normal appearing epithelia: a predictor of colorectal cancer metastasis? Int J Biol Sci 9: 119-133, 2013.

15. Lu Y, Jingyan G, Baorong S, et al: Expression of EGFR, Her2 predict lymph node metastasis (LNM)-associated metastasis in colorectal cancer. Cancer Biomark 11: 219-226, 2012.

16. Toiyama Y, Yasuda H, Saigusa S, et al: Increased expression of Slug and vimentin as novel predictive biomarkers for lymph node metastasis and poor prognosis in colorectal cancer. Carcinogenesis 34: 2548-2557, 2013. 